\title{
Urgences
}

\section{Journal de saxifrage}

\section{Sylvie Gagné}

Numéro 29, octobre 1990

\section{Éclats d'œuvre}

URI : https://id.erudit.org/iderudit/025597ar

DOI : https://doi.org/10.7202/025597ar

Aller au sommaire du numéro

\section{Éditeur(s)}

Urgences

\section{ISSN}

0226-9554 (imprimé)

1927-3924 (numérique)

Découvrir la revue

Citer ce document

Gagné, S. (1990). Journal de saxifrage. Urgences, (29), 8-25.

https://doi.org/10.7202/025597ar d'utilisation que vous pouvez consulter en ligne.

https://apropos.erudit.org/fr/usagers/politique-dutilisation/ 


\section{GEUVRES ET FRAGMENTS}




\section{Journal de saxifrage Sylvie Gagné}

pour Ariane et Renaud ces fragments qu'ils me laissent parfois écrire entre leurs larmes et leurs rires

Car enfin qu'est-ce que l'homme dans la nature? Un néant à l'égard de l'infini, un tout à l'égard du néant, un milieu entre rien et tout, infiniment éloigné de comprendre les extrêmes; la fin des choses et leurs principes sont pour lui invinciblement cachés dans un secret impénétrable.

Également - incapable de voir le néant d'où il est tiré et l'infini où il est englouti. Que fera-t-il sinon d'apercevoir quelque apparence du milieu des choses dans un désespoir éternel de connaître ni leur principe ni leur fin. Toutes choses sont sorties du néant et portées jusqu'à l'infini.

Pascal

Les ruines douées d'avenir, les ruines incohérentes avant que tu n'arrives, homme comblé, vont de leurs parcelles à ton amour.

René Char

\section{Note liminaire}

Entre l'infiniment grand et l'infiniment petit, la totalité et le fragment, la pupille se dilate, la vue s'abîme. Notre univers n'est-il qu'un fragment issu de la formidable explosion d'un système primitif? La totalité est-elle une illusion? Comme il semble impossible d'en faire le deuil. Peut-on la reconstituer, la restaurer?

Tout n'est-il pas toujours fragment de quelque chose? On fragmente ce qui nous est transmis. On ne retient, plus tard, 
que des fragments d'une histoire d'amour (la séduction se jouant sur quelques détails magnifiés). On n'écrit que des fragments de vie, de regards, de sensations, d'émotions. Du monde au mot et à nouveau au monde. Dans quel ordre rendre compte de tout cela?

On est toujours en continuité avec quelque chose, en filiation (réelle ou mythique), ne serait-ce que pour rompre. Rien de plus ambigu et déroutant que de n'avoir rien à briser pour se démarquer. On construit mal dans le vide, un peu mieux sur les décombres.

Le fragment est nécessaire; aucun discours ne peut, à lui seul, proposer une vue de l'ensemble hétéroclite des impressions de l'existence. Des fragments de plusieurs discours se rassemblent. Mais comment en faire la synthèse et est-ce toujours nécessaire?

N'y aurait-il que des fragments? Ne sommes-nous pas toujours à la recherche de pièces manquantes? Et le monde, la structure, l'encyclopédie, l'œuvre?

On pense toujours qu'à l'origine était un Tout. Sommesnous sans cesse en quête d'une unité perdue? Et les ruptures, l'inachèvement, les histoires lacunaires, les présences isolées autour desquelles brille l'absence?

Ne sommes-nous pas issus à la fois d'une conjonction et d'un processus de division? Notre vision du monde n'embrasset-elle pas les champs de la réalité autant avec un grandangulaire qu'au téléobjectif (sans jamais toutefois saisir fidèlement cette réalité)? Comme on le voit, le domaine de réflexion est on ne peut plus vaste.

La partie et le tout. Le fragment et l'œuvre. Le rapport de ces deux termes ne se pose pas sous la forme d'un dualisme radical. Un créateur n'est-il pas, à divers moments de son travail, tour à tour hanté par l'œuvre et obsédé par le fragment?

J'aimerais m'approcher de cette problématique par un tracé personnel et provisoire. Mon premier réflexe est toujours de ne pas m'autoriser à intervenir avant d'avoir " fantasmé" de tout lire sur la question ou presque. Je ne devrais pas me prononcer avant d'avoir maîtrisé les ouvres complètes (elle envie terriblement ceux qui arrivent à travailler indéfiniment sur une seule petite phrase - quoique 
s'il s'agit de celle de la sonate de Vinteuil, on frôle l'infini...). Je vois toujours d'abord un "abîme de science", pour reprendre l'expression de Gargantua. Cette véritable boulimie encyclopédique (plus modérément, elle préfêre quand même la culture générale à la fragmentation du savoir) s'avère être une bonne méthode pour retarder, voire même rendre impossible, le moment de l'écriture.

En ce moment, par exemple, j'aimerais bien, avant même de songer au fragment, me plonger dans la lecture des théoriciens de la "postmodernité" ou m'aventurer dans les dédales du concept d'œuvre tel qu'élaboré par les romantiques allemands pour soutenir leur adhésion à l'expression fragmentaire en opposition à l'œuvre finie. Ou encore relire, avec cet enthousiasme et ce plaisir sans cesse renouvelés, certains livres "fragmentaires" de Barthes ou de Blanchot. Mais je n'en ai, pour le moment, malheureusement ni le temps, ni les moyens. Alors... Le journal, forme fragmentaire et intermittente par excellence, est posé là, entrouvert... «Sous l'alibi de la dissertation détruite, on en vient à la pratique régulière du fragment; puis du fragment, on glisse au “journal".»1

1 Roland Barthes, Roland Barthes par Roland Barthes, coll. * Écrivains de toujours ", $n^{\circ}$ 96, Paris, Seuil, 1975, p. 99. 


\section{Jour A}

Saisir d'abord le mot «fragment". Le chercher en ce recueil privilégié de sédiments de signification, ce "trésor » de la langue: le dictionnaire. Juste après fragilité, fragment. Nous voici dans un champ sémantique où nous passons des "fragments d'un vase, d'une statue antique» aux fragments "du passé " ou "de la vérité". On souligne en chemin la "partie d'une œuvre dont l'essentiel a été perdu ou n'a pas été composé" de même que la "partie extraite d'une œuvre, d'un texte quelconque " (citation, extrait, passage). Ce qui est fragmentaire s'oppose à encyclopédique. Cela tient du débris, de la brisure, de l'ébauche, de la conception. C'est quelque chose d'incomplet, de partiel, de morcelé. Quant à la fragmentation, c'est l'action de partager en fragments, de concasser; mais c'est aussi, en biologie, la «fragmentation du chromosome, interrompant la succession des gènes, et provoquant une mutation " 2 . De là, on peut toujours rêver...

Si le fragment est postérieur à l'œuvre, si c'est tout ce qui reste d'une œuvre ancienne, on songe au travail archéologique. De l'œuvre en amont, ne nous parviennent que des fragments qui devraient servir à reconstituer des textes de l'Antiquité ou du Moyen Âge, par exemple. Le fragment tire son origine d'ailleurs; il est un descendant. On remonte du fragment à l'œuvre-mère.

De tels fragments conservent parfois leur mystère presque entier; d'une part, à cause de la discontinuité des morceaux de texte, voire l'absence même de l'essentiel; d'autre part, en raison du regard et de l'interprétation très fragmentaires (comme toujours) qui s'y posent, compte tenu de l'écart temporel, culturel, etc.

J'imagine aisément qu'on puisse être obsédé (y passer sa vie) par l'idée de reconstituer la totalité originelle d'un objet, d'un site, d'un événement, d'une histoire ou d'une "vérité". Ainsi en est-il sans doute des fragments recueillis lors de fouilles archéologiques (enfant, elle avait reçu un petit morceau de pierre ponce provenant supposément de Pompéi. Elle y tenait beaucoup.). On demande au fragment de montrer la voie vers toute une ville, une civilisation. 
Freud n'a-t-il pas succombé à une curiosité similaire? On connaît sa fascination pour l'archéologie. Il s'intéressait vivement aux fouilles de son époque et alla jusqu'à transposer le procédé à la technique psychanalytique; on y cherche les traces, les fragments d'un passé, d'une histoire individuelle: "Tout se passe comme si Schliemann avait de nouveau mis à jour cette ville de Troie que l'on croyait imaginaire. ${ }^{3}$

L'analyse n'est-elle pas un lieu privilégié pour entrevoir un impressionnant travail de fragmentation: celui à l'œuvre dans le rêve, dans l'activité fantasmatique, dans la déformation du souvenir, dans l'indifférence à l'égard de l'ordre chronologique, dans les déplacements ou dans l'interprétation même, pour ne mentionner que ces aspects. Ce que l'on arrive à reconstituer n'est pas une pièce authentique mais déjà une autre construction. On ne saura jamais toute l'histoire, toute la vérité. On apprend à vivre avec tous ces fragments faufilés.

Il y a d'ailleurs des fragments dont on ne rêverait jamais de reconstituer l'ensemble. Ainsi en est-il sans doute de ces petits morceaux de Mur de Berlin...

Mais un fragment peut également provenir d'une œuvre plus ou moins récente, disponible en sa version intégrale. C'est le cas de l'extrait (figurant dans une anthologie, par exemple) ou de la citation qui nous tombe sous les yeux ou au creux de l'oreille et qui provoque le goût de lire le texte en entier. De fil en aiguille, par le chas du fragment, je remonte à l'œuvre. Quelque chose me touche dans un passage et j'imagine pouvoir prolonger ce ravissement.

Dans l'œuvre que je lis, il y a de multiples percées du fragment. D'abord, ma lecture est souvent interrompue par des rêveries, des effets de connotations, autant de fragments qui reviennent s'insérer dans l'œuvre lue et se mêler à mes interprétations.

De plus, je peux chercher à lire le pluriel d'un texte et c'est ma lecture qui en fragmente la continuité; je peux, par la suite, tenter aussi d'en rendre compte de manière fragmentée.

3 Sigmund Freud, La naissance de la psychanalyse, Lettres a Wilhelm Fliess, notes et plans (1887-1902) [1956], publiés par Marie Bonaparte, Anna Freud et Ernst Kris, traduit de l'allemand par Anne Berman, Paris, PUF, 1969, p. 272. 
Des fragments de textes s'introduisent à leur tour dans mon écriture, soit par un phénomène d'intertextualité souvent assez diffus, soit par une citation spécifique que je choisis comme épigraphe (ce petit corps étranger qui oriente parfois étonnamment la lecture du texte auquel il a été greffé) ou comme insertion dans le corps du texte (par autorité ou purement par plaisir, par affinité). De la lecture d'autres textes, mon écriture est restée imprégnée.

Enfin, il m'arrive fréquemment de lire quelques passages de mes auteurs préférés avant d'écrire; il ne s'agit pas d'une documentation, mais d'un stimulant. J'aime en particulier les entrevues, sans doute parce que la pensée nous est livrée de manière fragmentaire. 
14

Jour B

Venons-en au fragment antérieur à l'œuvre; rien n'a véritablement été brisé (si ce n'est, peut-être, une certaine continuité intérieure... ce qui n'est pas rien). Le fragment est une sorte d'origine. L'œuvre est en aval, c'est ce vers quoi, en principe, on descend en suivant le courant. Le mouvement se ferait du fragment vers l'œuvre à venir (ou l'abîme). Le limon s'accumule, le texte se fait, le fragment engendrant une structure toujours différée (sur l'air de: Ça n'est pas encore ça). Feuilles volantes, notes éparses dont le destin est incertain. Vers quel genre de fragment m'entraînera-t-il ?

Tant qu'on ne parle pas d'agencement, d'organisation et même de genre, on est encore dans le fragment. Ce n'est que plus tard qu'il contribuera à un genre spécifique ou les contiendra tous en germe et se fera genre en soi (le fragment se ferait-il antidote au problème des genres en permettant à tous de survivre?).

Tant qu'on ne parle pas de structure, on est à pied d'œuvre. On est dans l'inquiétude et l'enthousiasme de la conception, du projet, de l'étincelle, du commencement, dans la saisie des flashes, le repérage. On est à la veille de faire prendre corps à ce quelque chose qui murmure. Le geste d'écrire n'est pas totalement posé. Ça se joue dans la prise de notes, de mots, de formules, mais le plus souvent mentalement, en monologues intérieurs. Une petite voix (le refrain avant l'opéra) souffle des idées, des bouts de phrases, des bribes de conversations imaginées, de très petites unités d'écriture qui ne se rendent souvent même pas sur papier, ou quand ils se rendent, ce ne sont plus que des fragments plus minces encore. C'est dans les transports en commun, sur la route ou avant de s'endormir que surgissent les fragments les plus * embrayeurs ».

Parfois, souvent même, tout s'arrête là, au seuil de l'élaboration secondaire, si l'on peut dire, juste avant la corvée de la composition. Tout s'arrête là, juste avant le travail de réalisation d'un produit susceptible d'être mené à terme, fini et publié.

Cela voudrait-il dire qu'il n'y a pas d'œuvre si l'on ne dépasse pas cette étape? Rejoindrait-on alors cette partie de la définition du fragment qui laisse entendre que l'essentiel n'a pas été composé? Rien de moins sûr. 
On imagine mal une œuvre sans fragments, soit qui l'ont précédée, avant sa composition définitive, soit qui la suivent, advenant une éventuelle fragmentation. L'œuvre toute blanche a besoin de toutes les couleurs des fragments.

Mais imagine-t-on un fragment sans œuvre? Tout dépend de la souplesse du concept d'œuvre. L'inachèvement est une apparence trompeuse. Les " œuvres vives" ne sontelles pas cette partie de la coque d'un navire qui se situe audessous de la ligne de flottaison? Le concept d'œuvre peut entrer en dilatation ou en mutation et le pluriel s'impose. 
16

\section{Jour C}

L'œuvre et le fragment s'affrontent si l'œuvre est synonyme de monument, de musée, de temple ou de mausolée, si elle cherche à revêtir un caractère définitif et à se présenter comme produit, résultat, chef-d'œuvre. L'opposition se calme si l'œuvre s'accepte plutôt comme échafaudage, chantier, atelier, qu'elle se fait mise au point temporaire, étonnement permanent et qu'elle se donne incessamment comme travail, production, main-d'œuvre. Son achèvement ne serait qu'une "formalité":

A un certain moment, les circonstances, c'est-à-dire l'histoire, sous la figure de l'éditeur, des exigences financières, des tâches sociales, prononcent cette fin qui manque, et l'artiste, rendu libre par un dénouement de pure contrainte, poursuit ailleurs l'inachevé. ${ }^{4}$

À son tour, le fragment, même s'il n'exclut pas les répétitions, l'ambivalence et parfois même certaines contradictions, ne doit pas sous-entendre le délire, lincohérence, l'incommunicabilité et encore le désœuvrement, la dissipation, l'accessoire, le superflu (quand le fragment fait saillie, qu'il est hors d'œuvre, s'il n'est pas de trop).

Il ne se compare pas à une version abrégée d'un phénomène plus considérable comme on opposerait, par exemple, un week-end de villégiature et un voyage autour du monde ou une aventure et une histoire d'amour. pète:

Le fragment est aussi doué d'une certaine force centri-

Un livre, même fragmentaire, a un centre qui l'attire; centre non pas fixe, mais qui se déplace par la pression du livre et les circonstances de sa composition. Centre fixe aussi, qui se déplace, s'il est véritable, en restant le même et en devenant toujours plus central, plus dérobé, plus incertain et plus impérieux. Celui qui écrit le livre l'écrit par désir, par ignorance de ce centre. ${ }^{5}$

En relisant ce passage de Blanchot, on ne peut s'empêcher de songer à la pratique du fragment évoquée par

4 Maurice Blanchot, L'espace littéraire, coll. « ldées", $n^{\circ} 155$, Paris, Gallimard, 1955, p. 10.

5 lbid., p. 5. 
Barthes: «Ecrire par fragments: les fragments sont alors des pierres sur le pourtour du cercle: je m'étale en rond: tout mon petit univers en miettes; au centre, quoi ?» 6

Cette stratégie de la dispersion du sujet, Barthes la signale toutefois comme illusoire; à travers cette figure du pluriel, subsiste un certain narcissisme incontournable: "J'ai l'illusion de croire qu'en brisant mon discours, je cesse de discourir imaginairement sur moi-même, [...] en croyant me disperser, je ne fais que regagner sagement le lit de l'imaginaire. " 7

Derrière le surgissement "désordonné " de tous les fragments, n'y a-t-il pas le rythme même d'un sujet qui s'écrit? 
18

\section{Jour D}

Curieusement, j'ai parfois l'impression que le fragment me confine à l'œuvre de " jeunesse * (petite, on la disait brisetout), comme si cette dispersion correspondait à un manque de maturité, comme si j'étais lasse du morcellement corporel que cela semble trahir, comme si tous les fragments étaient en quelque sorte des restes dont il faut se défaire. L'œuvre "rêvée * rétablirait l'essentiel, la continuité, la totalité, la perfection. Elle permettrait de contenir tous les morceaux, tous les objets partiels, et protégerait d'une menace de destruction.

Éternel souhait... quand je serai grande, j'écrirai une œuvre de longue haleine, monument interminable (un fragment plus long, quoi) qui me protégerait alors de quoi? De l'incontournable totalité de la mort? Ce n'est qu'à ce moment-là, d'ailleurs, que la mention " œuvres complètes " s'appose... même sur l'inachevé.

Lorsqu'on rêve d'une œuvre qui parviendrait à totaliser toutes les expériences, ne cherche-t-on pas à contrôler, par l'écriture, un trop-plein de stimulations? J'apprécie les détails (pas tout à fait synonymes de fragments) et je ne veux en perdre aucun. Je ne peux me résigner à quelque séparation que ce soit. L'angoisse provient du surplus. L'œuvre se fait collection, obsession.

$\mathrm{Si}$, au contraire, on traverse une période creuse, sans doute s'accroche-t-on aux rares matériaux qui s'offrent pour empêcher le vide de nous anéantir. Le fragment se fait mélancolie.

Parfois, il est un objet de transition satisfaisant en attendant l'œuvre ou le silence. 


\section{Jour E}

Je préfère habituellement le niveau de la mer aux abysses ou aux neiges éternelles des plus hauts sommets. Je suis plus familière avec le fragment et le texte qu'avec l'œuvre, terme plus inaccessible et terrifiant. D'ailleurs, on parle plus facilement de l'œuvre d'un autre que de la sienne. La vision que l'on a de sa propre écriture est essentiellement fragmentaire.

Si je ne sais parler de l'œuvre que j'écris (ce serait, entre autres, prétention de ma part), ce n'est toutefois pas nécessairement à cause d'une quelconque connotation négative rattachée au mot œuvre.

Que le fragment soit un terme qui me touche davantage relève, au départ, d'un aspect quantitatif. J'ai peu écrit et je considère dès lors ces textes davantage comme les fragments d'une œuvre virtuelle, en suspens, c'est-à-dire dont je ne sais si elle connaîtra une suite. J'ignore si d'autres fragments viendront s'additionner ou encore si des textes plus considérables viendront s'ajouter pour qu'un jour, tout cela puisse tenir dans une œuvre, c'est-à-dire une somme, un ensemble d'écrits d'une certaine ampleur.

Par ailleurs, le mot œuvre revêt un aspect qualitatif (voire mythique) qui me semble encore plus éloigné de mes fragments, surtout si l'on songe à des facteurs tels qu'un univers intérieur, une certaine "profondeur", un grand principe unificateur, un style, un rayonnement d'influence sur d'autres écritures ou individus, un engagement social, etc. Rien de tout cela dans mes balbutiements. Ce qui ne veut pas dire pour autant que je n'ai pas l'impression d'écrire... mais pas une œuvre.

Jai une écriture "circonstancielle", rarement quotidienne (sauf en temps de crise, et encore). Démarche sporadique dont le trajet comporte de fréquents arrêts. Écriture intermittente, conditionnelle, couverte d'aspérités ('’œuvre fantasmée est ronde et lisse comme un galet bien poli). L'œuvre que je rêverais d'écrire est en abîme, elle est de l'ordre du roman-fleuve. Mais je n'écris qu'au compte-gouttes.

Les œuvres qui me captivent sont souvent marquées par la suite (mais aussi par la répétition, la reprise rénovatrice de fragments). J'affectionne les briques... Je relis Le quatuor d'Alexandrie, La recherche du temps perdu, pour ne citer que celles-là. 
20

Je rêve de continuité. Ce qui ne m'empêche pas d'être fascinée par des cuvres plus fragmentaires comme celles de Barthes ou de Blanchot, pour ne nommer que ceux-là (c'est toujours à regret qu'elle se contente d'évoquer quelques éléments alors qu'elle songe à toute une série... autre symptôme encyclopédique...). Guvres fragmentaires mais totalement impressionnantes.

Le projet d'une œuvre monumentale empêche l'écriture. On renonce vite à écrire l'œuvre d'une vie (comme si c'était un objectif que l'on pouvait se fixer...). Il est plus invitant d'envisager l'œuvre sur le vif (c'est un peu le fragment), de jouer avec le mercure ou la limaille plutôt qu'avec l'acier. Si l'on se rappelle que le grand œuvre en alchimie consistait à changer le plomb en or, le fragment serait ce plomb dense, malléable, discret et indispensable. 


\section{Jour F}

Entre continuité et fragmentation, l'écriture cherche à louvoyer. Lorsqu'on écrit, même des fragments, on s'active autant à rétablir une certaine continuité qu'à retracer des morceaux. Des filiations se devinent; on suit toujours, consciemment ou non, les brisées de quelqu'un... et certains coups de crayon produisent des brisures, des modifications, des renouvellements qui distinguent une écriture de ses modèles.

Le fragment, en principe, implique l'interruption, évite le continu. Mais on peut écrire continuellement des fragments. L'écriture assure un certain suivi dans ce travail fragmenté. On ne poursuit peut-être pas le grand dessein d'une ceuvre à faire jusqu'au bout mais l'écriture du fragment fait quand même preuve de patience et même d'acharnement. La reprise joue avec l'interruption. On reprend toujours un peu là où on a laissé, même si c'est pour repartir vers d'autres horizons.

L'écriture ressemble parfois à une secousse sismique; mais elle se comporte aussi parfois comme le sismographe qui enregistre, par son tracé, non seulement le tremblement de terre mais une certaine continuité de l'activité de la croûte terrestre, l'ensemble de ses mouvements, si minimes soient-ils. 
22

\section{Jour G}

Fragile fragment. Les deux termes ont quelque chose à voir avec briser. Éclat de pierre tendre et tenace. Le fragment est effraction, bris de frontières et d'enfermement. Il est aussi infraction, non-respect d'une loi, celle de l'unité.

Désert du fragment. Carrière de l'œuvre. Écrits mouvants où l'on s'enlise. Le fragment ne sait pas. Il se plaît dans l'incertitude, la semi-obscurité, et réfléchit. Grain qui ébranle l'engrenage de l'œuvre (ce n'est malgré tout qu'une virgule qu'on peut, tout au plus, espérer insérer dans le cours de l'interminable livre du savoir, mais quand même).

Certains fragments sont semés sur le sable et restent sans lendemain. D'autres sont bâtis à chaux et à sable. Fragments durables pour rendre l'éphémère. Mémoire à désensabler, minutes à décaper avant de les coucher sur la page. Le marchand de sable passe et le rêve éveille l'orchestre de ses fragments.

Le fragment est une écriture nocturne (c'est de jour que surgit le drame de la structure, des liens et des transitions). Le fragment est étoile filante, conte d'une nuitée. Le fragment et la nuit servent aussi ceux et celles dont la profession n'est pas exclusivement celle d'écrivain et qui voient leur temps pour écrire réduit par toutes sortes d'autres occupations.

Le fragment suit également le rythme des enfants, ceux que l'on a et qui écrivent, sans crier gare, avec bonheur et insouciance, chagrins et affolements, de grands bouts de nos vies, littéralement par-dessus tous ces petits textes qui voient bien difficilement le jour. 
Jour $\mathbf{H}$

Peut-être y aurait-il lieu de tracer une sorte de typologie des fragments? Au départ, il y aurait déjà tout un univers théorique à survoler uniquement pour aborder le fragment comme stratégie; on songe à de multiples pratiques littéraires d'époques et de factures très différentes au sein desquelles le fragment a constitué un geste de rupture, une solution de continuité: la fragmentation romantique, l'inachèvement comme transgression de la structure du langage artistique, le fragment dans la problématique de la modernité, le refus du code social par une certaine avant-garde du $20^{\circ}$ siècle, le fragment dans le discours de la postmodernité, etc. (elle aime bien cette petite abréviation car elle pointe vers tout le reste). Encore un sujet de recherche en abîme. Envisageons, pour le moment, une plus modeste énumération.

Si, pour certains, le fragment est une stratégie, pour d'autres, c'est une limite personnelle: le fragment et pas plus. Il n'y a pas assez de souffle pour entreprendre la traversée de certains thèmes, ou l'on ne se sent pas suffisamment habité par une question. La curiosité est là, mais on ne peut s'approcher que brièvement.

Parfois, c'est le fragment tout simplement, parce qu'il convient. Certains sujets n'appellent pas de longs développements, ils se prêtent à un style lapidaire. On arrive à condenser, à mettre le doigt rapidement sur l'essentiel. $\mathrm{Ou}$ encore, un seul détail retient notre attention.

Il y a aussi le fragment par plaisir; on joue avec les pièces d'un casse-tête (l'analogie est plus forte si l'on songe à ceux dont l'objectif n'est pas de reconstituer une image mais simplement toute la surface d'une même couleur; il n'y a pas d'autre repère que la patience du joueur). Ou encore, on aime formuler des titres, des projets, des introductions; on reste dans les préliminaires: "Aimant à trouver, à écrire des débuts, il tend à multiplier ce plaisir: voilà pourquoi il écrit des fragments: autant de fragments, autant de débuts, autant de plaisirs. ${ }^{8}$ 
Jour I

C'est la dimension temporelle du fragment qui nous sollicite le plus. C'est souvent le temps qui impose le fragment avec plus ou moins d'urgence (la nature et le degré de l'urgence détermineraient le type de fragment).

Ainsi, en lisant les Feuillets d'Hypnos de René Char, nous sommes très profondément atteint par ce passage: "J'écris brièvement. Je ne puis guère m'absenter longtemps. S’étaler conduirait à l'obsession. " 9 Ces notes ayant été prises alors que Char se battait dans la Résistance (1943-1944), on imagine sans peine que la menace quotidienne de la mort laisse peu de temps pour une composition plus élaborée.

Dans un tout autre contexte (sans transition... comme disent souvent les présentateurs des nouvelles françaises télévisées), le temps intervient et suggère le fragment. Ce sont les Fragments d'un discours amoureux, pour reprendre le titre du très beau livre de Barthes, livre qui répond, en quelque sorte, au souhait de ce personnage de roman:

Entre l'éternité et le point, le temps des gens amoureux. On devrait l'écrire en fragments. La maxime n'est pas la rhétorique des logiciens concentrés. La maxime devrait être le langage de la passion, qui n'oublie rien mais n'a pas de temps à perdre. ${ }^{10}$

Le fragment peut aussi être une forme provisoire car, pour le moment, il ne peut en être autrement. On dit qu'on trouve toujours du temps pour faire ce que l'on désire intensément (ce n'est pas toujours vrai, mais passons). Il faut prendre du temps pour répondre à l'appel, à l'injonction de l'écriture.

Je trouve le temps pour le fragment mais non pour la réunification de tous ces premiers jets. J'aurais le temps pour le poème (dont les liens sont plus flottants, fondants) ou pour certaines formes d'essais, mais difficilement pour le roman. Ce dernier est l'objet d'un fantasme d'écriture très répandu auquel je n'échappe pas. C'est un roman dont je rêve comme d'une thèse dont je n'arriverais à écrire que des chapitres, dans un désordre insoutenable...

9 René Char, Fureur et mystère, coll. " NRF Poésie ", Paris, Gallimard, 1962, p. 94.

10 Julia Kristeva, Les Samourais, Paris, Fayard, 1990, p. 377. 


\section{Jour $\mathbf{J}$}

Le fragment rappelle le naufrage. L'écriture brisée. On peut flotter sur plusieurs petits écrits avant de s'ancrer. Le fragment serait nomade; il me tient en exil sur des îlots d'écriture dispersés autour du continent de l'œuvre potentielle. Mais la distance qui les en sépare représente parfois la mer à boire. Parfois aussi, on préfêre la solitude de cette écriture insulaire.

Le fragment s'apparente aux saxifrages, ces plantes qui poussent dans les rochers, les fissures des murs. L'écriture exerce une poussée et brise. Le fragment se glisse, traverse des blocs de connaissance, d'existence et ce, même s'il faut y aller par quatre chemins. Il avance souvent seul mais parvient, avec toutes ses variations, à envahir de vastes surfaces de travail.

En ce jour J, pas d'attaque ou de règlement final. Si le fragment multiplie en quelque sorte les entrées, il a bien du fil à retordre avec les conclusions. Le mot de la fin se fait attendre.

Ce texte s'est écrit au fil d'un été et s'interrompt avec lui, comme un journal de "vacance". Chacun de ces morceaux pourrait être repris (aurait même sans doute intérêt à l'être) avec plus de rigueur ou de souplesse, développé différemment comme un négatif agrandi avec plus ou moins de contrastes, orienté autrement comme les pièces d'un mobile ou présenté sous une forme plus achevée. Une autre fois... 